\title{
Fuzzy Inference Routing Algorithm to Enhance the Network Lifetime of Heterogeneous Wireless Sensor Networks
}

\author{
Muneeb Afzal C \\ Assistant Professor \\ Department of Electronics and Communication Engineering \\ Navodaya Institute of Technology, Raichur-584103, \\ Karnataka, India
}

\begin{abstract}
In the wireless sensor network system the route discovery and the dissemination of the node energy has been the major aspect in the network lifetime. The nodes in the network will be distributed at the area by using the searching method for the link or the path. All the nodes in a network are connected to each other by using the link establishment with the minimal energy utilization. Hence the distributed energy aware fuzzy logic assisted routing protocol has been proposed with the comparative study of fuzzy inference. The tabu search and the fuzzy assisted routing for data transmission have been proposed to compare and analyze the algorithms for better life time of the network.
\end{abstract}

Keywords—Fuzzy logic, tabu search, wireless sensor network

\section{INTRODUCTION}

In general, the use of the network has been in high need as many of the real time applications will be processed by the line services or the network node. The WSN or wireless sensor network system is capable of not only transmitting the data but also sensing the data arrival and transmission by using the specially designed sensors. [1] The use of sensor nodes is efficient to reach the optimal goal of data transmission.

In the recent time the increase in the lifetime of the WSN has been gaining the interest because of its low cost processing. Many methods like LEACH have been developed to increase the overall lifetime.

Problems causing low lifetime of network

- Looping node links

- Overlapping paths in the routes

- No data aggregation

- More nodes in unreachable state

- No energy aggregation etc

In this work we have stated the routing, overlapping and link distance based criteria to solve the problem.

$\mathrm{T}$ Haider et al, proposed an energy aware routing mechanism using the concept of the fuzzy logic. The system gateway will do the operation of arranging the routes for the nodes and also maintains the centralized sensor nodes routing table which will indicate that which of the hop is next to the other nodes [2]. It uses the fuzzy logic concept for determining the total cost of the established link between

Singh et al, surveyed many of the research works which are in general focused on mainly the energy efficient hierarchical sensor nodes cluster-based routing protocols in the architecture of the WSN. The main challenge in any of the proposed models was energy sources for the sensors in the data transmission. The design of protocols in the WSN has the

\author{
Dr. K Venkatachalam \\ Professor and Head \\ Department of Electronics and Communication \\ Navodaya Institute of Technology, Raichur-584103, \\ Karnataka, India
}

objective to keep the sensor nodes operating as long as possible, hence it will enhance the total network lifetime. [3]

$\mathrm{S}$ Al Shawi et al, proposed a routing method that extends the overall lifetime of the network by using the combination of fuzzy approach and also by using the unique A-star algorithm. The proposed method has been based on computation centrally of the routing schedule in view of the BS. [4]

$S$ Lee et al, proposed the fuzzy-logic based nodes clustering algorithm by using the energy predication model with it. Objective of proposed algorithm is to enhance the total lifetime of WSN. It is achieved by distributing the workload across the nodes evenly by the proper selection of the CHs. [5]

Tripti Sharma et al, proposed fuzzy based master cluster head election LEACH protocol. The selection of the $\mathrm{CH}$ is achieve by using the fuzzy rules set based on the total available energy and based on the proximity of the total distance need to traverse by using the selected path. The node having the residual energy among the remaining of the $\mathrm{CH}$ is selected as the Master Cluster Head (MCH). It is the responsible node for the transmission of the data by aggregating to base station. [6]

The main objectives of the proposed works are:

1. Increase the lifetime of the individual node and network

2. To make the link or path stable over period of time

3. Fuzzy network can be modeled into multiple networks

4. To achieve the same in heterogonous network in single architecture

\section{SYSTEM ANALYSIS}

\section{A. Existing system}

In the past, many models which has been used such as LEACH Artificial Bee Colony (ABC) which is inspired by the honey bees operation, Multi-hop Routing with LEACH (MRLEACH) protocol, Cost Function Based Routing Algorithms and many more of the methods has been proposed in the past by many authors having some issues as

Disadvantages

- $\quad$ Energy is not efficiently used

- Nodes cannot be stable longer time

- Lifetime of network is less

- Nodes link may be in loop 


\section{B. Proposed System}

Many methods are considered such as the energy required by using the node energy and packet rate to discover the network lifetime. Here the proposed algorithm is Fuzzy inference Routing System (FIRS) based distributed energy aware fuzzy logic protocol (F-DEFL) which will be compared with the existing Tabu searching for route establishment with the connected nodes. Reaching each node is important as it will keep the node data updated. The need of the connected nodes will help to utilize the energy as least as possible. The method will be compared with the existing methods like MDR [7], MTE [8], FA [9], DEFL [10] and the proposed F-DEFL.

\section{METHODOLOGY}

\section{A. Network topology}

The sensor nodes in the network have been distributed across the network topology of mesh. The use of the network topology has been adopted for the secure and reliable data transmission.

The topology will have the following conditions.

- The nodes number will be allowed to user to initialize and each of the nodes will be connected by using the shortest distance based system.

- The link between then nodes will be allowed not to overlap or loop.

- Nodes at uniform distance

- Should not form the loop

\section{B. Energy consumption model}

It's the total amount of the energy needed to transit the data between the node I to node j. [8]

$$
E_{t}(i j)=\beta_{1}+\beta_{2} d_{i j}^{4}
$$

Where, dij - distance between nodes,

$\beta 1=50 \mathrm{~nJ} / \mathrm{bit}$ - energy consumed in transmitter circuitry,

$\beta 2=100 \mathrm{pJ} / \mathrm{bit} / \mathrm{m}^{\wedge} 4$ - energy consumed at transmitter amplifier, here path loss exponent of 4 represents multipath reflection.

$\beta 3=150 \mathrm{~nJ} / \mathrm{bit}-$ the energy needed in receiving circuitry, a constant and is specified.

\section{Network initializations}

In this phase the (general) network with the number of nodes, fixed path and multiple networks will be initialized. The source (Src) and the destination (Dst) are prefixed in the system. In the network each node is assumed to be sensor node having sense of data arrival and departure and is denoted by $\mathrm{Si}$ for the ith sensor node. The remaining set of nodes are denoted as

$\mathrm{v}=\{\mathrm{v} 1, \mathrm{v} 2, \ldots, \mathrm{vN}\},,|\mathrm{v}|=\mathrm{N}\}$

The communication links or edges are denotes as

$\mathrm{E}=\{\mathrm{e} 1, \mathrm{e} 2, \ldots, \mathrm{eN})$

Neighbor: As we know network consist of number of nodes hence the neighbor nodes in the network are denoted as

$\mathrm{Vi}=\{\mathrm{i} \in \mathrm{N} \mid \mathrm{d}(\mathrm{Vi}, \mathrm{Vj}) \leq \mathrm{D}, \mathrm{n} \neq \mathrm{i}\}$

Where,

$\mathrm{N}=$ all nodes in network,
$\mathrm{d}(\mathrm{Vi}, \mathrm{Vj})=$ distance between each node $\mathrm{Vi}$, and $\mathrm{Vj}$,

$\mathrm{D}=$ Travelling distance

The cost for transmission of a k-bit packet or data over distance $\mathrm{D}$ is:

ETx $(\mathrm{k}, \mathrm{d})=\mathrm{k} *$ Eelec $+\mathrm{k} * \operatorname{\varepsilon fs} * \mathrm{~d} 2 \mathrm{~d}<\mathrm{d} 0(1)=\mathrm{k} *$ Eelec $+\mathrm{k}$

$* \operatorname{emp} * \mathrm{~d} 4$

$\mathrm{d}>=\mathrm{d} 0$

Where,

Eelec=base energy required to run the transmitter or receiver circuitry

$\varepsilon f$ s \& $\varepsilon m p=$ Energy of the transmitter amplifier

To receive the message, energy required is

$\operatorname{ERX}(\mathrm{k})=\mathrm{k} *$ Eelec

\section{Lifetime of sensor network}

Lifetime of the network is the time of the network will utilized in transmission of the data from one node to another or the source to destination nodes.

\section{E. Fuzzy logic}

Fuzzy logic represents the truth values of the variables which will be any of the possible type of the real number in between the range of the 0 and 1 . It's a classical deployment model of the system which is using the vector dataset for the tracing of the Boolean values [11].

The fuzzy logic has main four parts:

1. Rule base: it has the rule set of IF- THAN based on the rule data values

2. Fuzzification: convert inputs to fuzzy sets.

3. Inference Engine: current fuzzy input degree determined by each rule and it decides which are the rules fired based on the input field.

4. Defuzzification: convert the fuzzy sets in the inference engine to crisp value.

\section{F. Fuzzy inference routing system}

FIRS is a popular and well known routing protocol, [12] it includes the route table management in it to avoid the looping and node failure. The route table will help to decide the path of the data to be transferred. Each node will be having information of the neighbor nodes with the help of the routing table.

The complete FIRS algorithm can be expressed as follows:

1. Destination nodes IP Address

2. Destination nodes Sequence Number

3. Valid flag

4. Other state flags (e.g., available, unavailable, error)

5. Network Interface protocol (UDP)

6. Nodes Count

7. Next Hop

8. List of Precursors

In every route the nodes will be assigned the entry id. The FIRS uses the unique Id of each node to achieve the goal. The sequence number will be made available to the protocol during the built or routing table.

Nodes $(\mathrm{N})=\{\mathrm{n} 1(\mathrm{id} 1), \mathrm{n} 2(\mathrm{id} 2) \ldots . \mathrm{n}(\mathrm{idn})\}$

Before finalizing the route the FIRS performs the following sequence of message transmission operations like: 
1. Route Requests (RREQs)

2. Route Replies (RREPs)

3. Route Errors (RERRs)

The UDP will be receiving the messages known as sequence messages which will be used to check the availability of the nodes. Requests include the data type, data destination and the real time payload. The Request and the replies will be carried out in the sequence to confirm the node. If the node is available, than the route is selected and the operation is repeated till the destination if there are no errors found by the nodes.

\section{G. Fuzzy inference model}

The fuzzy based inference is the new technology compare to the classic neural network where the data set is manipulated based on the previous knowledge of the data. The fuzzy based inference performs the same operation as the neural network but in the detailed manner with the number of the iterations.

Fuzzy inference makes the grouping of the elements into the fuzzy assisted set and the true value is proposed by the surface and the edge values of it.

The fuzzy assisted class is defined as

$\sim \mathrm{C}=\{\mathrm{i} \mid \sim \Pi(\mathrm{i})\}$

Where,

$\sim \mathrm{C}$ : fuzzy set satisfying each individual i

$\sim \Pi$ : classification of the predicate of fuzzy a fuzzy propositional valued function.

$\sim \mathrm{P}(\mathrm{U}): \sim\{. \mid\}:. \mathrm{V} \times \mathrm{PF} ? \sim \mathrm{P}(\mathrm{U})$

Where,

$\sim\{. \mid$.$\} : it is in the domain in fuzzy class.$

$\mathrm{V}$ : set of the variables

$\sim$ PF: set of fuzzy propositional functions

$\sim \Pi . \mu: \sim \mathrm{PF} \times \mathrm{U} ? \sim \mathrm{T}$

Where,

$\sim \mathrm{T}$ : set of fuzzy truth variable of values (ranging 0 to 1 ).

Fuzzy propositional function an analogous expression which contains more than one value variable the assignment these variables make the expression to the fuzzy proposition based on the variables.

The fuzzy classification is combining or grouping the similar features variables together known as the fuzzy set. The fuzzy classification is the member function $\mu$ which indicates whether each of the individual is the unique member of the given class, in terms only if it is fuzzy classification based predicate

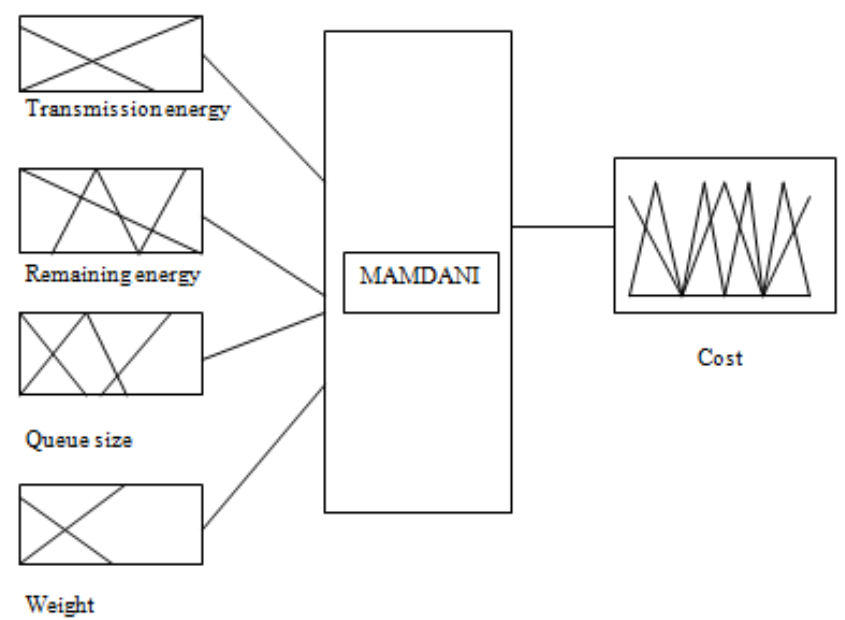

Figure.1.General Fuzzy Inference System

\section{H. Tabu search}

It is a type of the search engine for the data route based on the nodes availability. [13] It is a meta-heuristic that guides the local heuristic search the route of the solution in specific beyond local optimality.

Steps to consider while developing the tabu search engine

1. Design algorithm which returns initial solution,

2. Define moves to neighborhood $\mathrm{N}$ of the solution $\mathrm{s}$,

3. Determine content-size in the tabu lists,

4. Define the aspiration based criteria for search,

5. Design, intensification \& diversification based mechanisms.

\section{PROPOSED SYSTEM ARCHITECTURE}

The system architecture is shown in Figure 2. The proposed algorithm checks all the nodes present in the network and calculates the cost required to reach each node present in the network and checks the energy of all the nodes and updates the routing table. It uses tabu search to find the best path and energy of each node along with fuzzy logic. Finally the network lifetime of proposed algorithm is compared with various algorithms.

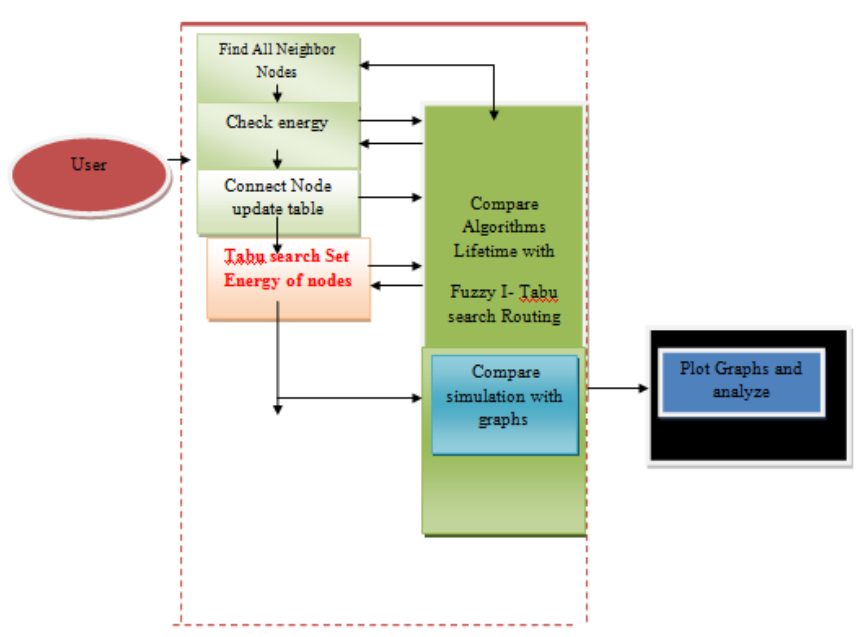

Figure.2. Proposed System Architecture. 

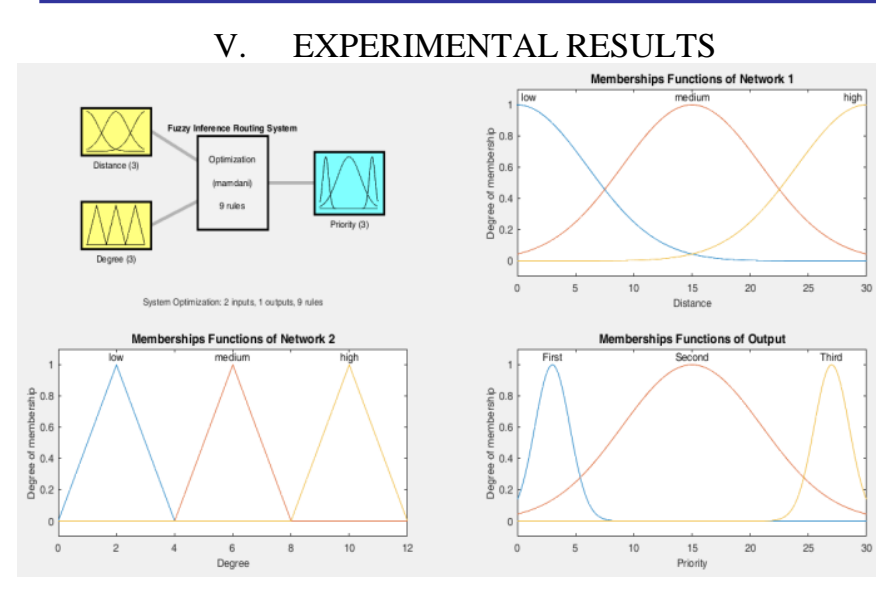

Figure.3.Fuzzy logic model and membership functions

Figure 3 shows the fuzzy logic model and its membership functions. The fuzzy rule base defined in this design consists 3 rules for each input hence there are total $3^{\wedge} 2=9$ number of rules

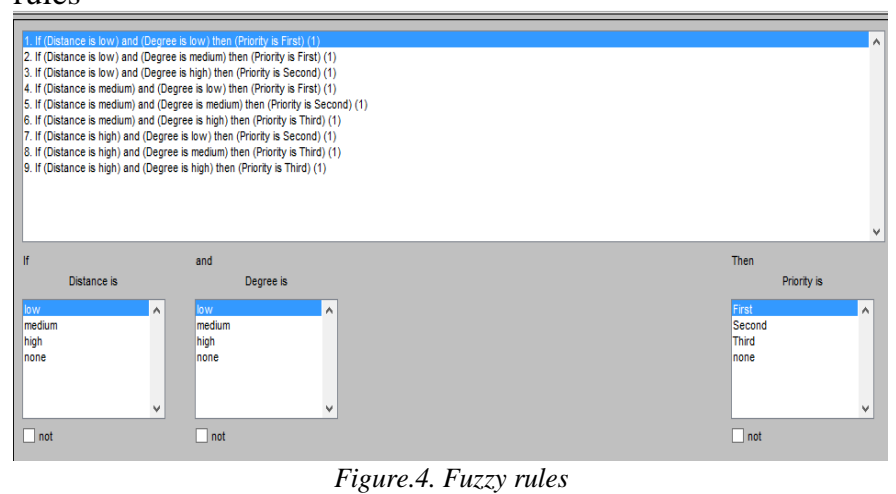

In Figure 4 IF-THEN rules are used in the proposed fuzzy system. As can be seen from Fig 8.4, human decision based logic is involved in the design. As an example, IF Distance is low and Degree is low, than priority is high. Here the fuzzy inference technique used is Mamdani method.

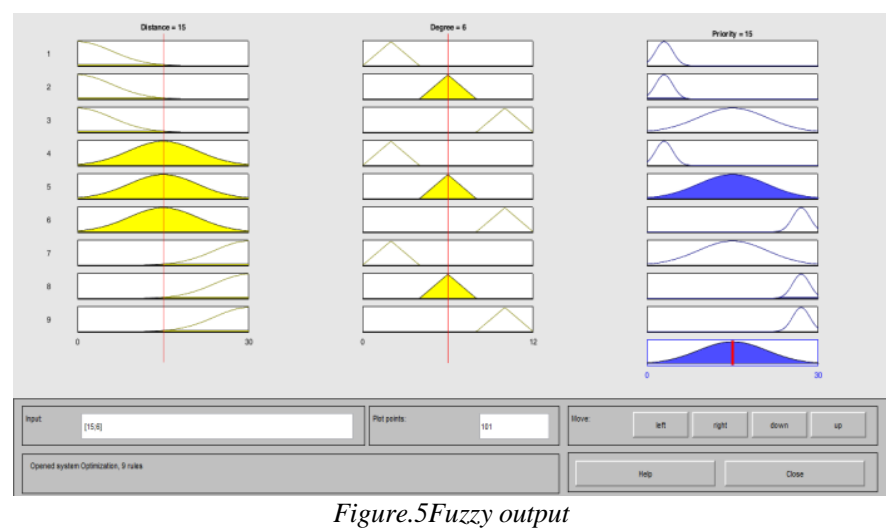

Figure 5 shows the fuzzy logic output, there are two inputs namely distance and degree, distance represents the distance of the nodes from sink node in a network and the degree represents the orientation of the nodes with respect to the sink node. All rules are processed by fuzzy inference engine.
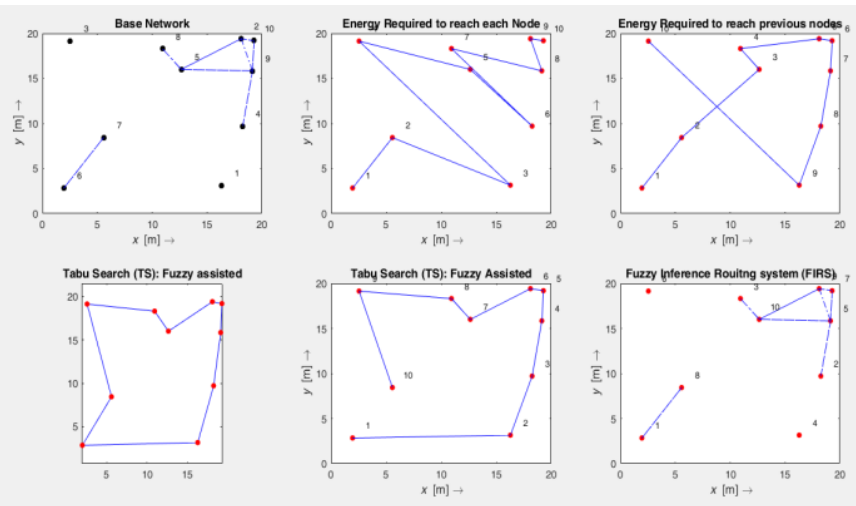

Figure.6. Network model showing nodes and routing scenario

Figure 6 shows the nodes and routing in a network; it uses fuzzy inference system along with tabu search to calculate the best cost to reach the neighboring node. For e.g. the cost to reach the neighboring node in base network is 6 but with FIRS and tabu search the cost is reduced to 1. The Fig 8.5 also shows the energy required to reach each node and previous node, the tabu search traces all the possible paths and selects the best possible path with best and least cost and then it is given to FIRS.

The below Figure 7 shows the graphical comparison between Flow Augmentation (FA), Minimum Drain Rate (MDR), Minimum Time Energy (MTE), DEFL and proposed F-DEFL. Compared to all the methods it is found that the proposed algorithm F-DEFL consumes less energy, which in turn increases the lifetime of the network.
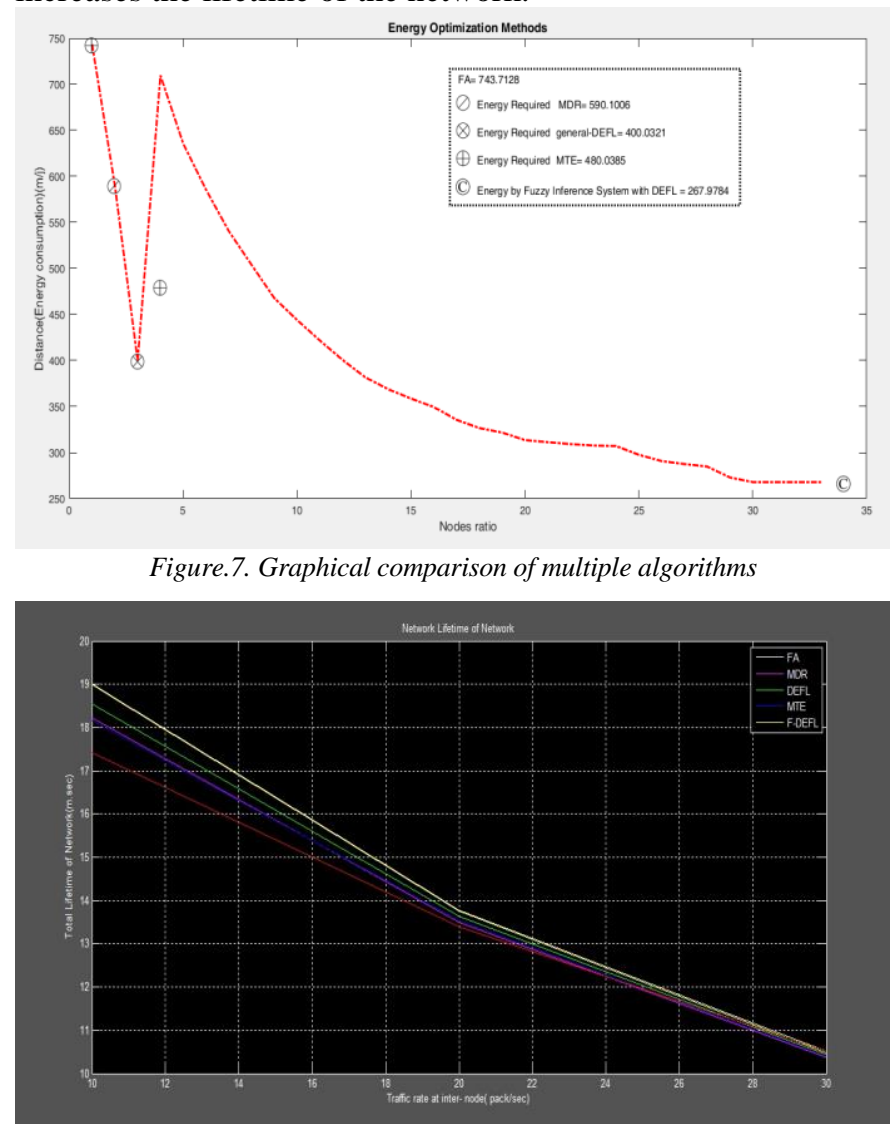

Figure.8. Graphical analysis of network lifetime for multiple algorithms 
Figure 8 shows total network lifetime vs. the traffic rate at inter node, it is found that the proposed algorithm has the maximum network lifetime has been compared to all other algorithms, MTE shows poor network lifetime performance. In the network but in case of the FA algorithm it has been shows good performance, however FA algorithm is more theoretical and complex and difficult to implement.

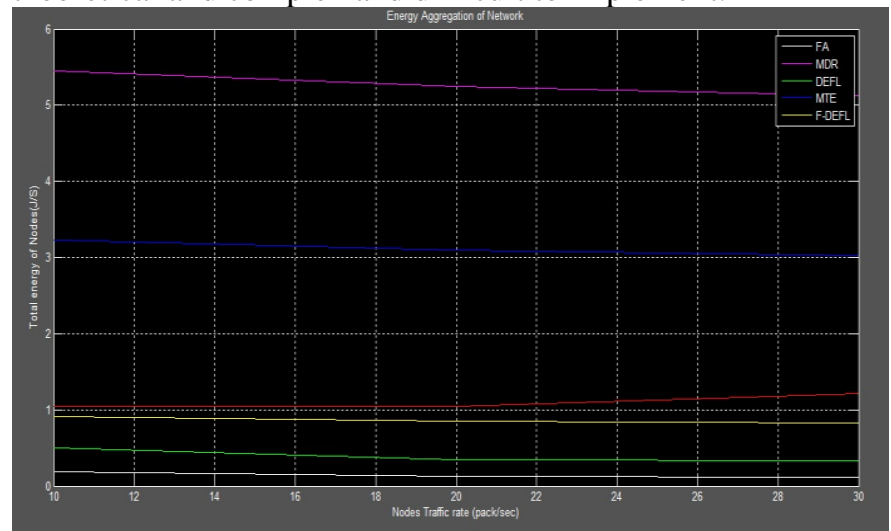

Figure.9. Graphical analysis of energy aggregation for multiple algorithms

Figure 9 shows total energy of the nodes vs. nodes traffic rates of various algorithms, it is found that MDR consumes more energy followed by MTE. FA consumes less amount of energy compared to all. Proposed algorithm F-DEFL consumes the average energy

\section{V1. CONCLUSION}

In this work the lifetime of the WSN is enhanced by the comparative study of the fuzzy inference model and the present methodologies. The proposed work demonstrates the use of tabu searching and fuzzy assisted searching of the nodes distance and routing. The factor which effects the network lifetime is the cost of data transmission which is based on the nodes distance and the connectivity of the links. The proposed work will analyze the concept of low cost utilization by using the DEFL method. The proposed work F-
DEFL has been compared in the simulation of MATLAB with the FA, MTE, MDR and DEFL.

\section{REFERENCES}

[1] T. Rault, A. Bouabdallah, and Y. Challal, "Energy efficiency in wireless sensor networks: A top-down survey," Computer Networks, Vol 67, 2014, pp 104-122.

[2] T. Haider and M. Yusuf, “A fuzzy approach to energy optimized routing for wireless sensor networks.” Int. Arab J. Inf. Technol., Vol 6, 2009, pp 179-185.

[3] Singh et al, "A survey on swarm intelligence based routing protocols in wireless sensor networks", International Journal of Physical Sciences, Vol 5,2010, pp 2118-2126.

[4] S. AlShawi, L. Yan, W. Pan, and B. Luo, "Lifetime enhancement in wireless sensor networks using fuzzy approach and a-star algorithm,' IEEE Sensors journal, Vol. 12, 2012, pp 3010-3018.

[5] S Lee and Cheng, "Fuzzy-logic-based clustering approach for WSNs using energy predication IEEE sensors journal', Vol 12, Sep 2012, pp 2891-2897.

[6] Tripti Sharma, Brijesh Kumar, "F-MCHEL: Fuzzy Based Master Cluster Head Election Leach Protocol in Wireless Sensor Network", International Journal of Computer Science and Telecommunications, Vol 3, October 2012, pp 8-13.

[7] D. Kim, J. Garcia-Luna-Aceves, K. Obraczka, J.-C. Cano, and P. Man- zoni, "Routing mechanisms for mobile ad hoc networks based on the energy drain rate," IEEE Transactions on Mobile Computing, Vol 2, 2003, pp 161-173.

[8] V. Rodoplu and T. H. Meng, "Minimum energy mobile wireless networks," IEEE Journal on selected areas in communications, vol. 17 1999, pp 1333-1344

[9] J.-H. Chang and L. Tassiulas, "Maximum lifetime routing in wireless sensor networks," IEEE/ACM Transactions on networking, Vol 12, 2004, pp 609-619.

[10] Al-Kiyumi et al.'Fuzzy Logic-based Routing Algorithm for Lifetime Enhancement in Heterogeneous Wireless Sensor Networks", IEEE Transactions on Green Communications and Networking, 2018, pp 2473-2400.

[11] "Fuzzy Logic". Stanford Encyclopedia of Philosophy. Bryant University. 2006-07-23. Retrieved 2008-09-30.

[12] Pedro Henrique Gouvea Coelho et al."Application of Fuzzy Inference Systems in the Transmission of Wireless Senso Networks", International Conference on Enterprise Information Systems, Vol 1, 2017, pp 618-624.

[13] Orojloo and Haghighat. "A Tabu search based routing algorithm for wireless sensor networks" Springer sciences, 2015.

[14] Fred Glover. "Tabu Search-Part 1" OSRA Journal on Computing1989, pp 190-206. 